\title{
Entwicklung von Schutzkonzepten gegen (sexuelle) Gewalt im medizinisch- therapeutischen Bereich
}

\author{
Ulrike Hoffmann', Jörg M. Fegert , Elisa König ${ }^{1}$, Anna Maier ${ }^{1}$ und Maik Herberhold ${ }^{2}$ \\ ${ }^{1}$ Klinik für Kinder- und Jugendpsychiatrie/Psychotherapie, Universitätsklinikum Ulm \\ ${ }^{2}$ Praxis für Kinder- und Jugendpsychiatrie und -psychotherapie Bochum-Langendreer
}

\begin{abstract}
Zusammenfassung: Theoretischer Hintergrund: Bekannt gewordene Fälle von (sexuellen) Übergriffen in Institutionen sowie Befragungsergebnisse zeigen, dass es auch in medizinischen Einrichtungen Fälle von Gewalt gegen Kinder und Jugendliche gibt. Fragestellung: Im Kontext von Krankenbehandlung liegen systemische Risikofaktoren für Übergriffe vor. Es ist deshalb wichtig, dass sich medizinische Einrichtungen mit dieser Problematik auseinandersetzen und Schutzkonzepte entwickeln. Methode: Es wird ein Überblick über Risikofaktoren sowie die Elemente von Schutzkonzepten gegeben. Ergebnisse: Ein Schutzkonzept ist ein System von Maßnahmen, die für einen besseren Schutz vor (sexuellen) Übergriffen in der Institution sorgen. Diskussion und Schlussfolgerung: Es ist notwendig, dass es in Institutionen eine klare Haltung gegen (sexuelle) Gewalt gibt. Die Erarbeitung eines Schutzkonzeptes trägt zur (Weiter-) Entwicklung dieser Haltung bei und erhöht das Sicherheitsgefühl der Fachkräfte zum Vorgehen im konkreten Fall.
\end{abstract}

Schlüsselwörter: institutioneller Kinderschutz, Schutzkonzepte, Kindesmisshandlung, sexueller Missbrauch, medizinische Einrichtungen

Development of Safeguarding Measures Against (Sexual) Abuse in the Medical-Therapeutic Field

Abstract: Background: Institutions in the (mental) health sector serving children play an important role in protecting children by (1) treating assaulted children and adolescents and (2) offering them a safe environment where they are protected from assaults within the medicaltherapeutic institution. Reported cases of (sexual) assault in institutions as well as the results of surveys illustrate that violence against children and adolescents does take place in institutions of (mental) health care. A more detailed examination of the cases reveals that systemic risk factors exist for (sexual) assaults in the context of medical and therapeutic treatment. This perspective is often neglected in the self-image of health institutions as it contradicts their self-perception as places where people can get help and treatment. Objective: Facilities of (mental) health must deal with this problem and develop safeguarding measures. This paper provides an overview of possible risk factors in the medical-therapeutic field, presenting elements of safeguarding measures and providing advice on their practical implementation. Methods: Safeguarding measures aim at reducing risk factors, preventing assault, and fostering a safe environment. They comprise structures, standards, and procedures enabling the recognition, identification, and prevention of assaults against children. Standards for safeguarding measures are defined by central committees, but each institution should specifically approve and adopt those standards based on an analysis of the risk and potential within the institution, thereby identifying situations and procedures that pose a high risk of (sexual) assault. Studies show that safeguarding measures have not yet been comprehensively implemented in medical-therapeutic institutions. Discussion and Conclusion: Examples of elements to be included in safeguarding measures are a mission statement, behavioral guidelines, a pedagogical concept, and a concrete intervention plan for dealing with cases of sexual abuse. Risk factors may be present in various areas, such as the specific situations of the patients in care (e. g., children and adolescents with mental illnesses) and the medicaltherapeutic interventions (e.g., physical examination, psychotherapy), but also in problematic institutional structures (e.g., lack of staff, insufficient professional competences, lack of participation). Even though the development of safeguarding measures requires time and resources, it bears many advantages for institutions, such as an increased sense of security for the professionals, too, because they gain knowledge about how to proceed in a specific case. Safeguarding measures were incorporated into the guidelines of quality management of the German Federal Joint Committee, making their implementation obligatory for institutions of health care. Accordingly, all institutions must now deal with providing protection against sexual abuse and violence in the institution and developing appropriate concepts.

Keywords: child protection in institutions, protection concepts, safeguarding measures, training, child abuse, child sexual abuse, medicaltherapeutic institutions 
(Sexuelle) Übergriffe auf Kinder und Jugendliche in Institutionen des medizinisch-therapeutischen Kontextes sind ein relativ häufiges Problem (siehe Beitrag Rassenhofer, Korger, Fegert \& Hoffmann in diesem Heft). Bei genauerer Betrachtung bekannt gewordener Fälle wird deutlich, dass es im Kontext einer Krankenbehandlung oder Therapie systemische Gefährdungsfaktoren für (sexuelle) Übergriffe gibt. Medizinische Institutionen müssen deshalb ein Schutzort für die ihnen anvertrauten Patient_innen sein. Dies bedeutet, dass institutionelle Strukturen und Abläufe so gestaltet sind, dass Grenzüberschreitungen und (sexuelle) Gewalt erkannt, benannt und Maßnahmen ergriffen werden, diese zu stoppen bzw. präventiv zu verhindern. Zudem haben medizinische Institutionen im Kinderschutz auch eine wichtige Funktion, da viele von Gewalt und Missbrauch betroffene Kinder und Jugendliche irgendwann mit dem medizinischen System in Kontakt kommen. Deshalb müssen medizinische Institutionen auch Kompetenzorte sein. Gemeint ist damit, dass Kinder und Jugendliche in medizinischen Einrichtungen kompetente Hilfe und Unterstützung finden und ein Ort gestaltet wird, am dem sie es schaffen können, sich anzuvertrauen (Kappler, Hornfeck, Pooch, Kindler \& Tremel, 2019).

Notwendig ist, dass sich medizinische Einrichtungen mit der Gesamtproblematik auseinandersetzen und sogenannte Schutzkonzepte entwickeln. Ein Schutzkonzept kann definiert werden als ein System von spezifischen Maßnahmen, die für den besseren Schutz von Mädchen und Jungen vor sexuellem Missbrauch und Gewalt in einer Institution sorgen. Schutzkonzepte sind als „Zusammenspiel aus Analyse, strukturellen Veränderungen, Vereinbarungen und Absprachen sowie Haltung und Kultur einer Organisation“ zu sehen (UBSKM, 2021). Ziel der Entwicklung von Schutzkonzepten ist es, die Rechte von Kindern und Jugendlichen zu stärken, Handlungsspielräume von Tätern und Täterinnen einzuschränken und die Handlungssicherheit der Fachkräfte zu erhöhen.

Der nachfolgende Beitrag gibt einen Überblick über Gefährdungsfaktoren im medizinischen Bereich, stellt die Elemente von Schutzkonzepten vor und gibt Hinweise zur praktischen Umsetzung in medizinisch-therapeutischen Institutionen, mit dem Fokus des stationären und ambulanten kinder- und jugendpsychiatrischen und pädiatrischen Bereichs.

\section{Entwicklung der Thematik „Schutzkonzepte im medizinischen Bereich"}

Diskussionen zur Entwicklung von Schutzmaßnahmen vor (sexueller) Gewalt in medizinischen Institutionen gab es bereits seit den 1990er Jahren, bekannt wurde zum Beispiel 1998 der Fall eines Chefarztes in der Kinder- und Jugendpsychiatrie in Holzminden, der über Jahre hinweg ihm anvertraute Kinder und Jugendliche sexuell missbraucht hatte. Dieser Fall wurde 1999 auf einer Sitzung der „Bundesarbeitsgemeinschaft Leitender Ärzte in der Kinder- und Jugendpsychiatrie" ausführlich diskutiert. 2004 wurde durch die Ethikkommission der drei Fachgesellschaften für Kinder- und Jugendpsychiatrie und Psychotherapie ein Papier zu körperlichen Untersuchungen konsentiert (Ethikkommission der drei Fachgesellschaften für Kinder- und Jugendpsychiatrie und Psychotherapie, 2004).

Im Nachgang des Holzmindener Falles führte Jörg M. Fegert gemeinsam mit der Pädagogin Mechthild Wolff das erste Projekt in Deutschland zur Problematik von sexuellem Missbrauch in Institutionen durch und veröffentlichte gemeinsam mit ihr ein Fachbuch (Fegert \& Wolff, 2002; 2006), in welchem Element von Schutzmaßnahmen in Institutionen skizziert und Vorschläge zur Umsetzung gemacht wurden.

Der genannte Fall führte jedoch nicht dazu, dass sich kinder- und jugendpsychiatrische und -psychotherapeutische Institutionen (und auch generell medizinische Institutionen oder andere Institutionen wie Heime etc.) nachfolgend flächendeckend mit der Thematik möglicher (sexueller) Übergriffe auseinandersetzten und Maßnahmen zur Prävention etablierten. Solche Entwicklungen blieben vielmehr Einzelfälle, die stark mit dem individuellen Engagement einzelner Fachkräfte oder Einrichtungen für die Thematik verknüpft waren.

Eine umfassende Beschäftigung mit der Thematik sexueller Übergriffe in Institutionen erfolgte erst wieder durch den sogenannten „Missbrauchsskandal 2010“, in dessen Kontext insbesondere Fälle im Bereich der katholischen Kirche und pädagogischer Einrichtungen bekannt und nachfolgend auch diskutiert wurden. In der Folge des Missbrauchsskandals wurde ein Runder Tisch „Sexueller Kindesmissbrauch" (RTKM) gegründet und die ehemalige Bundesministerin Dr. Christine Bergmann zur ersten Unabhängigen Beauftragten zur Aufarbeitung des sexuellen Kindesmissbrauchs (UBSKM) ernannt. Auch wenn die berichteten Fälle in dieser Zeit überwiegend aus dem pädagogischen und/oder kirchlichen Bereich stammten, wurden im Rahmen der Begleitforschung für die Unabhängige Beauftragte auch eine kleine Anzahl von Über- 
griffen aus dem medizinisch-therapeutischen Kontext berichtet. Aus diesen Fällen konnten Hinweise auf gefahrgeneigte Situationen im medizinisch-therapeutischen Bereich und Täterstrategien abgeleitet werden (Fegert, Rassenhofer, Schneider, Spröber \& Seitz, 2013).

Der Runde Tisch empfahl seinerzeit in seinem $\mathrm{Ab}$ schlussbericht allen Institutionen, in denen Kinder und Jugendliche betreut werden, Schutzkonzepte zu etablieren und formulierte Elemente, die einen Rahmen für die Inhalte des Schutzkonzeptes vorgeben, jedoch von jeder Institution spezifisch mit Inhalt gefüllt, angepasst und umgesetzt werden müssen (Bundesministerium für Justiz, Bundesministerium für Familie, Senioren, Frauen und Jugend \& Bundesministerium für Bildung und Forschung, 2012).

Seit 2010 haben sich diverse Einrichtungen auf den Weg gemacht, Schutzkonzepte zu entwickeln. In einer vom USBKM in Auftrag gegebenen Untersuchung zum Stand der Umsetzung von Schutzkonzepten in verschiedenen Bereichen hatte das Deutsche Jugendinstitut (DJI) hierzu eine Evaluation durchgeführt (Kappler et al., 2019). Die Ergebnisse zeigten, dass die Umsetzung in medizinischen Einrichtungen geringer ausgeprägt war als in pädagogischen Einrichtungen. Konkret wurde nach der Umsetzung verschiedener Elemente von Schutzkonzepten gefragt. Von den 165 Kliniken, die an der Befragung teilgenommen haben, gaben nur $15.8 \%$ an, alle abgefragten neun Bestandteile umgesetzt zu haben. Die Praxen wurden mit einem angepassten Instrument von insgesamt fünf Elementen befragt, so dass die Ergebnisse mit denen der Kliniken nicht direkt vergleichbar sind. Hier hatten nur $4,7 \%$ alle fünf abgefragten Elemente umgesetzt.

Im Vergleich zwischen Kliniken und Praxen zeigte sich also insgesamt ein höherer Umsetzungsgrad in Kliniken. Zudem lag insbesondere in den Praxen der Schwerpunkt auf der Intervention bei einem Verdacht auf Kindeswohlgefährdung bei den Kindern und Jugendlichen, die sich als Patient_innen in der Praxis vorstellen und war nicht auf einen möglichen Übergriff in der Einrichtung gerichtet.

Aus den Ergebnissen lässt sich ableiten, dass die Thematik im medizinisch-therapeutischen Bereich noch nicht umfassend angekommen ist. Deutlich wurde aus der Untersuchung weiterhin, dass die Entwicklung sowie die konkrete Umsetzung eines Schutzkonzeptes im Arbeitsalltag in allen Institutionstypen mit einem hohen Einsatz von personellen und finanziellen Ressourcen verbunden und deshalb schwierig zu leisten ist, und dass die Einrichtungen einen Unterstützungsbedarf bei der Entwicklung von Schutzkonzepten haben.

Bis zum vergangenen Jahr war die Entwicklung und Umsetzung von Schutzkonzepten in medizinischen Einrichtungen nicht verbindlich vorgeschrieben. Auf einen
Antrag der Deutschen Krankenhausgesellschaft (DKG) wurde jedoch im Jahr 2020 in den Gremien des Gemeinsamen Bundesausschusses (G-BA) über die Aufnahme der verbindlichen Entwicklung von Schutzkonzepten in die Qualitätsmanagement-Richtlinien (QM-RL) beraten und am 16.07.2020 eine Ergänzung beschlossen: „Einrichtungen, die Kinder und Jugendliche versorgen, müssen sich gezielt mit der Prävention von und Intervention bei (sexueller) Gewalt und Missbrauch (Risiko- und Gefährdungsanalyse) befassen. Daraus werden der Größe und Organisationsform der Einrichtung entsprechend, konkrete Schritte und Maßnahmen abgeleitet (Schutzkonzept).“ (G-BA, 2020, S. 1).

Somit müssen sich nun alle medizinischen Einrichtungen mit der Entwicklung und Umsetzung von Schutzkonzepten befassen.

\section{Aufbau von Schutzkonzepten}

Wie bereits ausgeführt hatte der RTKM in seinem Abschlussbericht Elemente eines Schutzkonzeptes definiert. Diese Elemente lassen sich den Ebenen Analyse, Prävention, Intervention und Aufarbeitung zuordnen (siehe nachfolgende Tabelle 1).

Die hier vorgegebenen Elemente bilden einen Rahmen, der jedoch von jeder Institution spezifisch inhaltlich gefüllt und angepasst werden muss. Notwendig ist dies, da jeder Arbeitsbereich (medizinisch, therapeutisch, pädagogisch etc.) spezifische Risikofaktoren für (sexualisierte) Gewalt aufweist und auch die Strukturen in Institutionen unterschiedlich sind (in der Medizin z. B. stationärer und ambulanter Bereich), was sich z. B. auf die Gestaltung eines konkreten Handlungsplan bei Übergriffen auswirkt.

Da die Diskussion um die Ausgestaltung von Schutzkonzepten sehr stark vom RTKM geformt und inhaltlich gefüllt wurde, liegt der Fokus auf Kindern und Jugendlichen und der Misshandlungsform des sexuellen Missbrauchs. Gerade im medizinischen Bereich sollten jedoch auch andere Misshandlungsformen (z. B. körperliche Gewalt) und auch andere Gruppen schutzbefohlener Menschen mitgedacht werden. Zudem gibt es im medizinischtherapeutischen Bereich viele unterschiedliche Berufsgruppen mit unterschiedlichen Tätigkeitsfeldern und Arbeitsbereichen und entsprechend unterschiedlichen Berührungspunkten mit den Patient_innen. Dementsprechend sind die Gefährdungsfaktoren für (sexuelle) Übergriffe unterschiedlicher und vielfältiger, als dies im pädagogischen Bereich der Fall ist (siehe hierzu auch Fegert, Allroggen \& Schloz, 2017). Auch das ist bei der Entwicklung des Schutzkonzeptes zu bedenken. 
Tabelle 1. Ebenen und Elemente von Schutzkonzepten (adaptiert nach UBSKM, 2021)

\begin{tabular}{|c|c|}
\hline Ebenen & Elemente von Schutzkonzepten \\
\hline Analyse & $\begin{array}{l}\text { - Gefährdungsanalyse } \\
\text { - Potentialanalyse }\end{array}$ \\
\hline Prävention & $\begin{array}{l}\text { - Präventionsangebote für die Kinder und Jugendlichen } \\
\text { - Pädagogisches, sexualpädagogisches und medienpädagogisches Konzept } \\
\text { - Kinderschutzsensible Personalrekrutierung und -entwicklung } \\
\text { - Arbeitsvertragliche Regelungen, z. B. Einholung eines Erweiterten Führungszeugnisses } \\
\text { - Berücksichtigung von Kriterien des Kinderschutzes in der Personalauswahl } \\
\text { - Regelmäßige Qualifizierung der Mitarbeitenden } \\
\text { - Leitbild } \\
\text { - Verhaltenskodex/Verhaltensleitlinien } \\
\text { - Partizipationsformen für Kinder und Jugendliche, Eltern und Mitarbeitende } \\
\text { - Konzept zum Management von Beschwerden und Anregungen }\end{array}$ \\
\hline Intervention & $\begin{array}{l}\text { - Interventionsplan zum Umgang mit Fehlverhalten von Mitarbeitenden } \\
\text { Mit dem Begriff Fehlverhalten sind hier umfasst: fachliches Fehlverhalten, (sexuelle) Grenzverletzungen, (sexuelle) Übergriffe/ } \\
\text { (sexuelle) Gewalt }\end{array}$ \\
\hline Aufarbeitung & $\begin{array}{l}\text { - Handlungsempfehlungen zum Umgang mit der Aufarbeitung aufgetretener Fälle } \\
\text { - Konzept zur Rehabilitation nach Falschbeschuldigung }\end{array}$ \\
\hline
\end{tabular}

\section{Grundsätzliches zur Umsetzung von Schutzkonzepten}

Um ein Schutzkonzept gegen (sexualisierte) Gewalt so in einer Einrichtung umzusetzen, dass es auch in der alltäglichen Arbeit gelebt wird, sind verschiedene Aspekte zu beachten.

Haltungsentwicklung: Es ist notwendig, in der Institution eine Haltung zu entwickeln, dass Übergriffe, gleich welcher Art und gleich gegen wen (also z.B. auch gegen die Mitarbeitenden), nicht geduldet werden. Im Zentrum dieser Haltung sollten die Achtung vor den Rechten und der Würde jedes Einzelnen stehen. Diese Haltung muss von den Leitungskräften aller Ebenen vorgelebt werden (Kölch \& Fegert, 2018a).

Prozesshaftigkeit der Schutzkonzeptentwicklung: Die Entwicklung eines Schutzkonzeptes muss als fortlaufender Prozess gedacht und konzipiert werden, in welchem es immer wieder Anpassungen und Verbesserungen geben muss. Es ist deshalb sinnvoll, eher von einem Schutzprozess als von einem Schutzkonzept zu sprechen, oder dies zumindest in diesem Sinne zu denken (Fegert, Schröer \& Wolff, 2017).

Partizipative Erarbeitung und Umsetzung: Die Elemente von Schutzkonzepten sollten, wo immer es möglich ist, in einem partizipativen Prozess gemeinsam mit den Mitarbeitenden und den Patient_innen entwickelt werden. Nur wenn Beteiligung an der Schutzkonzeptentwicklung stattfindet, ist der Grundstock dafür gelegt, dass ein Schutzkonzept auch im Alltag gelebt wird. Wenn Partizipation nur zum Teil möglich ist, müssen alle Betroffenen gut über die Ergebnisse der Schutzkonzeptentwicklung infor- miert werden (Rau \& Liebhardt, 2018; Fegert, Allrogen \& Schloz, 2017).

Institution als Schutzort und Kompetenzort: Wie bereits ausgeführt, hatte die Evaluation zum Stand der Umsetzung von Schutzkonzepten durch das DJI gezeigt, dass medizinische Einrichtungen die Unterstützung für im familiären Kontext betroffene Kinder und Jugendliche (also „externe Fälle“) sehr viel stärker im Blick haben, als Gefährdungen für Übergriffe in der Institution. Insofern muss überprüft werden, ob beide Perspektiven im Schutzkonzept berücksichtigt sind.

Leitungsverantwortung und Engagement stärken: Schutzkonzeptentwicklung braucht Mitarbeitende, die sich dafür engagieren. Die Aufrechterhaltung dieses Engagements ist nicht möglich, ohne dass dafür Ressourcen bereitgestellt werden. Hierfür muss die Leitungsebene einer Einrichtung sorgen. Diese hat ebenso die Aufgabe, den Prozess der Schutzkonzeptentwicklung in Gang zu bringen, anzuleiten, zu moderieren und die Mitarbeitenden, die sich hier engagieren, zu unterstützen (Fegert, Kölch \& Kliemann, 2018).

Offene und positive Fehlerkultur: Gemeint ist damit, dass Fehler und fachliches Fehlverhalten offen angesprochen werden können und Fehler als Möglichkeit gesehen werden, zu lernen und sich weiterzuentwickeln. Bei der Analyse von Fehlern gilt es, grundlegende Prinzipien der Fehlerentstehung zu erkennen, wie z.B. begünstigende Abläufe oder Strukturen, um die Wiederholung von Fehlern zu vermeiden. Grundsätzlich sollten nicht nur gravierende Fehler angesprochen und reglementiert werden, sondern auch niedrigschwelliges Fehlverhalten in der täglichen Arbeit. Wenn es auch die Möglichkeit gibt, dieses anzusprechen, nachzubearbeiten und Verbesserungen 
einzuführen, kann das dazu beitragen, dass auch gravierendes Fehlverhalten, wie etwa sexuelle oder körperliche Übergriffe, gemeldet wird. Gerade diese Fehlerkultur des offenen Ansprechens ist jedoch häufig schwierig umzusetzen, weil Personen, die Fehler melden, z. B. als Verräter_innen ihrer Kolleg_innen angesehen werden. Notwendig ist deshalb ein Beschwerdesystem in der Einrichtung, welches eine niedrigschwellige Möglichkeit gibt zu melden und dem auch vertraut wird (Kölch \& Fegert, 2018b).

Gewalt hat viele Ursachen: Gerade physische und psychische Gewalt gegen Patient_innen oder Vernachlässigung, z.B. von Pflegemaßnahmen, haben auch Gründe, die struktureller Natur sind (Hoffmann \& Fegert, 2018; siehe hierzu auch den Beitrag Fegert, Clemens \& Hoffmann in diesem Heft). Schutzkonzepte müssen auch solche Aspekte in den Blick nehmen und hier Abhilfe schaffen.

\section{Hinweise zur praktischen Umsetzung von Schutzkonzepten}

Am Anfang einer Schutzkonzeptentwicklung müssen eine Gefährdungs- und eine Potentialanalyse stehen. Ziel der Gefährdungsanalyse ist es, die spezifischen Gefährdungsfaktoren für (sexuelle) Übergriffe in der Einrichtung zu bestimmen, um nachfolgend passende Schutzmaßnahmen zu etablieren, die die Gefährdungsfaktoren eliminieren oder minimieren. Die Potentialanalyse hat das Ziel, Potentiale der Einrichtung in Bezug auf die Umsetzung eines Schutzkonzeptes, wie z.B. bereits bestehende Maßnahmen, sowie die Vor- und Nachteile für die Institution, zu analysieren.

Es kann gar nicht genug betont werden, dass bereits die Durchführung einer Gefährdungs- und Potentialanalyse eine erhebliche präventive Wirkung entfaltet, da im Idealfall alle Beteiligten in die Analyse eingebunden werden und somit neben der Informationsgewinnung auch ein institutionelles „Bewusstsein“ für Kinderschutz etabliert wird. Dieses kann bei jedem Einzelnen Aufmerksamkeit und Handlungssicherheit erzeugen.

Viele Institutionen haben bereits Elemente eines Schutzkonzeptes in der Institution etabliert, dies ist zum Teil jedoch nicht unter dem Label „Kinderschutz“ oder „Schutzkonzept" eingeführt. Wenn dies der Fall ist, ist es sinnvoll, sich das jeweilige Element nochmal unter dem Gesichtspunkt des Kinderschutzes anzuschauen.

Die Gefährdungsfaktoren, die im Rahmen einer Gefährdungsanalyse überprüft werden sollten, können folgendermaßen aufgegliedert werden (adaptiert nach König, et al., 2018):
1) Zielgruppe: Es ist danach zu schauen, ob in der Institution bestimmte besonders vulnerable Gruppen versorgt oder betreut werden, wie z.B. Kinder und Jugendliche oder Patient_innen mit psychischen Erkrankungen.

2) Personal/Personalpolitik: Gerade in Bezug auf personell-strukturelle Gefährdungsaspekte entfaltet sich die präventive Funktion einer fundierten Gefährdungsanalyse. $\mathrm{Zu}$ achten ist z. B. auf unzureichende Personalschlüssel, Probleme personeller Kontinuität, ungenügend fundierte Einarbeitung, unklare und/oder intransparente Definierung von Kompetenzen und Arbeitsaufgaben und fehlende Fort- und Weiterbildungsangebote. Eine Herausforderung für die Ermittlung der Gefährdungsaspekte stellen auch die unterschiedlichen professionellen Hintergründe und Aufgabenfelder der Mitarbeitenden aus Pflege und Therapie dar. $\mathrm{Zu}$ berücksichtigen ist in allen Einrichtungen auch das nicht-therapeutische Personal (Fahrdienst, Reinigungskräfte etc.).

3) Betreuungsverhältnis: $\mathrm{Zu}$ analysieren sind die spezifischen Settings, in denen die Patient_innen betreut werden und die sich in Kliniken und Praxen zum Teil gravierend unterscheiden (z.B. unbeaufsichtigte Einzelkontakte mit Patient_innen (Nachtdienst etc.), körperliche Untersuchungen). $\mathrm{Zu}$ beachten sind in besonderem Maße Interventionen, die ein hohes Gefährdungspotential haben, zur Machtausübung missbraucht zu werden, wie etwa freiheitsentziehende Maßnahmen und Zwangsmaßnahmen, sowie besondere Vertrauensbeziehungen, wie sie etwa in der Psychotherapie oder durch jahrelange Begleitung durch Hausärzt_innen entstehen können.

4) Fehlerkultur, Information, Beteiligungsstrukturen und Beschwerdemanagement: Gefährdende Faktoren aus dieser Gruppe sind z. B. unklare Zuständigkeiten, intransparente Entscheidungs- und Kommunikationswege, Informationsdefizite bei Patient_innen, mangelnde Einbindung von Eltern/Bezugspersonen in den Behandlungs-/Therapieprozess, ausgeprägte Hierarchien und Abhängigkeitsverhältnisse zwischen den Mitarbeitenden, keine Regeln für Umgang mit Fehlverhalten und kein etabliertes Beschwerdemanagement.

Bei der Gefährdungsanalyse sollte nicht nur der Kontext der Gefährdung von Patient_innen durch Fachkräfte (Ärzt_innen, Pflegekräfte etc.) betrachtet werden, sondern auch alle anderen Konstellationen von Übergriffen. Die dadurch ermöglichte Wirkung auf das Sicherheitsgefühl und ein auch in schwierigen (z.B. konfliktiven) Situationen angemessenes Verhalten der Fachkräfte wirkt sich reziprok unmittelbar auf eine Reduktion von Gefährdungssituationen von Patient_innen aus.

Die Gefährdungen, Unsicherheiten und Risiken müssen gemeinsam mit den Mitarbeitenden der Institution und den Kindern und Jugendlichen erkannt und bewertet 
werden (partizipativer Ansatz, s.o.). Nur dann wird sich ein umfassendes Bild ergeben.

Für die Durchführung einer Potentialanalyse kann das Verfahren der SWOT-Analyse genutzt werden. SWOT steht hierbei für Strengths $=$ Stärken, Weaknesses = Schwächen, Opportunities $=$ Chancen und Threats $=$ Risiken.

Fragestellungen sind in der SWOT-Analyse zum Beispiel (angepasst nach König et al. 2018, S. 458):

- Strengths = Stärken: Welche Maßnahmen zum Kinderschutz haben wir bereits umgesetzt? Welche Gefährdungsfaktoren konnten hierdurch reduziert werden?

- Weaknesses = Schwächen: Was läuft in Bezug auf Kinderschutz noch nicht so gut in unserer Einrichtung? Welche Maßnahmen sollten noch umgesetzt werden?

- Opportunities = Chancen: Welche positiven Aspekte bringt die Umsetzung von Schutzmaßnahmen für unsere Einrichtung mit sich?

- Threats = Risiken: Was sind Risiken/Probleme in Bezug auf die Umsetzung von Schutzmaßnahmen in unserer Einrichtung?

Auf Basis der Ergebnisse der Gefährdungs- und Potentialanalyse sollten dann die in Tabelle 1 aufgeführten Elemente von Schutzkonzepten umgesetzt werden. Es gibt bereits Fachliteratur, Fortbildungen und Beispiele aus der Praxis, an denen man sich bei der Umsetzung orientieren kann (z.B. Horvay \& Naumann, 2018; Fegert, Kölch, König et al., 2018; Universitätsklinikum des Saarlandes 2020; UBSKM, 2021; Online-Kurs „Leitungswissen Kinderschutz in Institutionen“ (https://leitung.elear ning-kinderschutz.de/)).

Nachfolgend werden einzelne Elemente des Schutzkonzeptes noch näher ausgeführt.

Wie bereits beschrieben, ist für die Etablierung eines Schutzkonzeptes die Entwicklung einer gemeinsamen Haltung notwendig, die Handlungssicherheit und -möglichkeiten auch in risikobehafteten und hoch emotionalisierten Situationen ermöglichen kann. Diese Haltung, sowie Ideen und Grundsätze einer Einrichtung sollten in einem Leitbild verschriftlicht werden, um nach innen Klarheit und Sicherheit zu vermitteln und sie prägnant nach außen kommunizieren zu können.

Ziel von Verhaltensleitlinien ist es, die in einem Leitbild ausgeführten grundsätzlichen Aussagen in konkretem gewünschtem Verhalten der Mitarbeitenden zu beschreiben, die Haltung einer Einrichtung also auf die Verhaltensebene zu übertragen. Diese Regeln sollten Prämissen widerspiegeln, wie die Autonomie der anvertrauten Personen so weit wie möglich zu respektieren, die Intimsphäre der Betreuten zu schützen, sie zu empowern und ihre Selbstpflegemöglichkeiten zu stärken (Fegert, Hoffmann \& Kölch, 2018). In einer Verhaltensleitlinie sollten auch die in der Gefährdungsanalyse festgestellten Risikosituationen aufgegriffen werden. Hilfreich ist auch, Wissen über Täterstrategien zur Anbahnung und Aufrechterhaltung sexueller Übergriffe zu haben, um auch diese Aspekte in die Verhaltensleitlinien einzubringen.

Verhaltensleitlinien schaffen durch klare Vorgaben Orientierung und Handlungssicherheit sowohl für die Mitarbeitenden als auch für die Leitungskräfte. Indem erwünschtes Verhalten explizit formuliert wird, ist es auch einfacher, dem/der Mitarbeitenden (fachliches) Fehlverhalten rückzumelden. Auf die Frage, welches Verhalten angemessen und erwünscht ist, gibt es nicht immer einfache Antworten. Vielmehr ist es notwendig, Verhaltensweisen und Abläufe zu diskutieren und zu reflektieren. (Kölch \& Fegert, 2018b).

Im Kontext der Entwicklung von Verhaltensleitlinien kommen immer wieder Diskussionen über das Thema Nähe und Distanz auf. Diese Thematik oft anhand von Konstellationen, wie körperliche Untersuchungen oder Handlungen/Behandlungen, in denen Kinder/Jugendliche sich mit einer erwachsenen Person allein in einem Raum befinden, besprochen. Es muss klar sein, dass medizinische oder therapeutische Arbeit ohne solche Interventionen nicht möglich ist und diese in dem Sinne Alltag sind. Keinesfalls sollten notwendige Untersuchungen vermieden werden, weil Unsicherheit oder Angst bestehen, Grenzen zu überschreiten. Es sind praxisnahe Lösungen zu suchen, die die Rechte der Kinder und Jugendlichen achten und schützen, gleichzeitig aber Diagnostik und Therapie ermöglichen (Fegert, Hoffmann \& König, 2020).

Um Kindern und Jugendlichen bzw. Patient_innen allgemein die Möglichkeit zu geben, Kritik und Beschwerden zu äußern, ist die Etablierung eines niedrigschwelligen, vertraulichen und altersadäquaten Beschwerde- und Anregungsmanagements notwendig (Rau \& Liebhardt, 2018). Wichtig ist, eine Option zu finden, die von der Zielgruppe angenommen und genutzt wird. Hierfür gibt es diverse Optionen, z. B. persönliche Ansprache (mit direktem Zugang zur nächsthöheren „Hierarchieebene“ oder in Praxen direkt der Praxisinhaber_in), Sprechstunde, Telefon, Email, Messenger-Dienste, Beschwerdeformular, Kummerkasten, Besuche einer/eines Patient_innenfürsprechenden auf Station, Gegensprechanlage auf Station, Hilfehotlines, Hilfeportale etc. Sinnvoll ist auch die Einrichtung einer externen Beschwerdestelle oder (z.B. für Praxen) der explizite Hinweis auf eine solche Stelle, so dass Beschwerden nicht einfach „unter den Tisch gekehrt" werden können. Deutlich sollte gemacht werden, was mit der Beschwerde passiert, wer darauf Zugriff hat und wie die dort geäußerten Aspekte weiterverarbeitet werden, z.B. Eingang in konkrete Verbesserungen finden. Sehr positive Wirkung entfaltet eine 
Rückmeldung an den/die Beschwerdeführer_in, nachdem im Team über die Beschwerde gesprochen wurde. Das Beschwerdesystem sollte ein breites Verständnis von Beschwerden haben. Nur wenn auch Anliegen des Alltags (z.B. Probleme mit Terminvereinbarungen, Gefühle des "Nichternstgenommenwerdens" durch Sekretär_innen oder Therapeut_innen, Klinikessen, Zimmergestaltung) als legitime Beschwerde in den Diskussionsprozess aufgenommen werden, entsteht das nötige Vertrauen in das Beschwerdesystem, sich auch bei schwerwiegenden Angelegenheiten, wie etwa sexuellen Übergriffen, zu trauen, eine Beschwerde zu tätigen. Es wäre also nicht zielführend, das Thema Beschwerden auf sexuelle Gewalt oder andere ähnlich gravierende Vorfälle zu begrenzen.

Zur allgemeinen Qualitätssicherung sollten auch systematische Befragungen zur Behandlungszufriedenheit gehören.

Um (Verdachts-)Fälle zu bearbeiten, ist die Erstellung eines Interventionsplans notwendig. Ziel der hier definierten Abläufe sollte es sein, einen Verdacht schnell zu klären, ggf. Gewalthandlungen zu beendigen, die Betroffenen zu schützen und angemessene Hilfsangebote für alle Beteiligten bereitzustellen (Winter \& Wolff, 2018). Wichtig ist es, sowohl die unterschiedlichen möglichen Konstellationen von Übergriffen zu durchdenken (s.o.), als auch die verschiedenen möglichen Szenarien, die Ergebnisse eines Falles sein können: Verdacht bewahrheitet sich, Verdacht lässt sich weder verifizieren noch falsifizieren, Verdacht stellt sich als Falschbeschuldigung heraus.

Es muss im Interventionsplan klar definiert werden, wer wann zu informieren ist. Unbedingt zu empfehlen ist die Einbindung externer Fachberatung, dies gilt in besonderem Maße für ambulante Praxen, die naturgemäß einen kleineren Organisationsgrad als stationäre Einrichtungen aufweisen. Ein frühzeitiger Einbezug externer Fachkräfte kann auch wirksam einem möglichen Anschein von Vertuschung oder „Betriebsblindheit“ entgegenwirken. Insofern kommt der vorausschauenden Kooperation mit Fachberatungsstellen und Kinderschutzambulanzen bereits im Vorfeld konkreter Vorfälle eine wichtige Bedeutung zu.

Institutionsspezifische Handlungsempfehlungen zur Aufarbeitung aufgetretener Fälle sollten anlassvorgreifend erarbeitet werden. Dies sorgt grundsätzlich für eine Sicherheit gebende Struktur und kann im Ereignisfall durch Aufarbeitung Erkenntnisse für die Optimierung bestehender Abläufe und Maßnahmen im Schutzkonzept ableitbar machen (Enders \& Schlingmann, 2018).

\section{Evaluation der Wirksamkeit von Schutzkonzepten und Weiterentwicklung}

Wie oben ausgeführt, wurde die Diskussion um die Notwendigkeit der Umsetzung von Schutzkonzepten und den Aufbau dieser maßgeblich vom RTKM und seither durch die Aktivitäten des UBSKM gesetzt und gerahmt. Die in diesen Kontexten formulierten Elemente sind bis auf Spezifizierungen unverändert geblieben und wurden auch nie infrage gestellt, sie bildeten die Grundlage der Evaluation des DJI (s.o.) und sind nun auch Gerüst der Vorgaben für die Umsetzung in medizinischen Einrichtungen durch den G-BA.

Aus Sicht der Autor_innen dieses Beitrages bilden die definierten Elemente generell die relevanten Aspekte ab, notwendig ist jedoch, wie auch der Runde Tisch schon betonte, die spezifische Ausarbeitung für den Kontext, in dem das Schutzkonzept angewendet werden soll. In diesem Falle würde es also darum gehen, Schutzkonzepte medizinisch zu denken. Beispiele sind die spezifischen Risikosituationen in medizinischen Einrichtungen (s.o.) und die Ermittlung nochmal spezifisch gefährdeter Gruppen (z. B. in der Kinder- und Jugendpsychiatrie sind viele Patient_innen, die bereits traumatische Erfahrungen gemacht haben und ein erhöhtes Risiko für eine erneute Traumatisierung haben). Auch werden sich etwa Interventionspläne und die Möglichkeiten der Partizipation im Vergleich zu z.B. pädagogischen Institutionen unterscheiden, aber eben auch ,innermedizinisch“ zwischen Kliniken und Praxen, zwischen Kinder- und Jugendmedizin und Kinder- und Jugendpsychiatrie.

Bisher nur in Ansätzen geklärt ist die Frage, wie Schutzkonzepte evaluiert werden können - also was macht ein Schutzkonzept zu einem guten Schutzkonzept? Für einige Elemente lässt sich dies anhand der Nutzung feststellen. Sicherlich ist zum Beispiel ein Beschwerdesystem dann gut, wenn Kinder und Jugendliche damit erreicht werden, wenn sie sich trauen, darüber eine Beschwerde zu tätigen. Auch für einen Interventionsplan wird man sagen können, ob er hilfreich und praktikabel ist, wenn er im Falle eines Falles erfolgreich angewendet werden konnte. Für andere Elemente ist nicht so einfach festzustellen, ob sie in der umgesetzten Weise gut und sinnvoll sind. Was macht zum Beispiel ein Leitbild zu einem guten Leitbild? Sicher trägt eine partizipative Erstellung dazu bei, jedoch macht auch diese keine Aussage darüber, ob die dort formulierten Aspekte im Alltag der Institution gelebt werden. Für die Einschätzung sind dann doch weniger die konkreten Elemente in ihrer Schriftform wichtig, als vielmehr die Haltung und das Klima in der Institution und der persönliche Einsatz der Mitarbeiten- 
den für die Thematik. Ein Indikator für diese Aspekte wäre, ob die Kinder und Jugendlichen sich in der Einrichtung wohl und sicher fühlen, ob sie sich eingebunden fühlen in Entscheidungen, ob sie sich gehört fühlen mit Anliegen und auch Beschwerden. Hier kann zur Evaluation z. B. das Tool „Du bist gefragt“ des USBKM genutzt werden (Webseite: https://fragen-an-dich.de/).

Ein Stück weit ist Evaluation auch in der anzustrebenden Prozesshaftigkeit der Schutzkonzeptentwicklung angelegt. Konkret wäre es ja das Ziel, Elemente von Schutzkonzepten umzusetzen, diese kontinuierlich auf ihre Funktionalität und Praxistauglichkeit zu überprüfen und weiterzuentwickeln. Auch hier gibt es jedoch keine etablierten Instrumente und Methoden, sondern Haltung und Einsatz der Leitung und der Mitarbeitenden werden im besten Fall für eine Optimierung sorgen.

In diesem Sinne ist die Eingliederung des Themas Schutzkonzeptentwicklung in das Qualitätsmanagement medizinischer Einrichtungen auch mit einer gewissen Skepsis zu sehen. Als positiv zu bewerten ist, dass die Thematik von Schutz vor Gewalt ein Stück weit zu einem „normalen“ Bestandteil von Qualitätsentwicklung wird, also die Nischenrolle und die Möglichkeit der Abwälzung auf sogenannte Missbrauchsbeauftragte verlässt. Ein Risiko ist aber, dass das Schutzkonzept als etwas betrachtet wird, was im Rahmen der Zertifizierung der Einrichtung erstellt und abhakt wird, und dann als Konzept im Schrank steht, ohne dass es im Alltag gelebt wird. Da die Regelung des G-BA noch relativ neu ist, wird erst im Verlauf sichtbar werden, wie sich die Entwicklung hier gestaltet.

\section{Zusammenfassung und Fazit}

Medizinische Einrichtungen haben im Bereich des Kinderschutzes eine wichtige Funktion, da viele von Misshandlung und sexuellem Missbrauch betroffene Kinder mit dem medizinischen System in Kontakt kommen. Daten sowie konkrete Fälle zeigen aber auch, dass medizinische Einrichtungen systemische Gefährdungsfaktoren für (sexuelle) Übergriffe auf Kinder und Jugendliche aufweisen. Es ist notwendig, dass sich medizinische Institutionen mit diesem Gefährdungspotential auseinandersetzen und Schutzkonzepte entwickeln, also Strukturen und Maßnahmen, um die Gefahr solcher Übergriffe zu reduzieren und im Fall eines Übergriffs angemessen zu reagieren. Zentral ist hierbei die Entwicklung einer Haltung in der Institution, dass Übergriffe, gleich welcher Art, nicht geduldet werden. Im Vorfeld möglicher Ereignisse etablierte Kooperation mit Fachberatungsstellen, festgelegte Handlungsabläufe und eine transparente Fehlerkul- tur geben Orientierung und Sicherheit. Schutzkonzepte sollten hierbei nicht nur auf sexuelle Übergriffe fokussiert, sondern breit gedacht und auf den Schutz aller Patient_innen in Institutionen sowie der Fachkräfte vor allen Formen von Gewalt ausgerichtet werden. Neben dem Schutz der Kinder und Jugendlichen vor (sexualisierter) Gewalt in der Einrichtung (Perspektive "Schutzort") sollte auch die kompetente Betreuung von gewaltbetroffenen Kindern und Jugendlichen, die wegen Auffälligkeiten oder zur Behandlung in die Einrichtung kommen, in den Blick genommen werden (Perspektive „Kompetenzort“).

Für die inhaltliche Gestaltung von Schutzkonzepten hat der RTKM Elemente definiert, die jedoch von jeder Einrichtung entsprechend der konkreten Voraussetzungen vor Ort mit Inhalt gefüllt werden müssen. Die Entwicklung eines Schutzkonzeptes ist dabei nicht als einmaliger Arbeitsschritt anzulegen, sondern ein Schutzkonzept sollte in einem fortlaufenden Prozess immer wieder weiterentwickelt und auf seine Umsetzung in der Praxis überprüft werden.

Mit der Verankerung in der QM-Richtlinie des G-BA ist die Entwicklung von Schutzkonzepten für medizinische Einrichtungen nun auch verpflichtend. Hierbei ist es eine erhebliche Herausforderung, unter Berücksichtigung der jeweiligen institutionellen Struktur und personellen Ausstattung eine prozesshafte Schutzkonzeptentwicklung zu etablieren. Dies fordert institutionsspezifische Lösungsmodelle, Diskussions-, Reflexions- und Entscheidungsprozesse, die in einer inhaberfokussierten Praxis mit einer überschaubaren Anzahl von Mitarbeiter_innen sicher anders aussehen wird, als in der ungleich größeren Personal- und Organisationsstruktur einer Klinik.

Noch weitgehend ungelöst ist die Frage der Evaluation von Schutzkonzepten. Hier fehlen bisher standardisierte Instrumente um die Qualität und Wirksamkeit von Schutzkonzepten zu belegen und diese systematisch weiterzuentwickeln.

\section{Literatur}

Bundesministerium für Justiz, Bundesministerium für Familie, Senioren, Frauen und Jugend, Bundesministerium für Bildung und Forschung (Hrsg.). (2012). Abschlussbericht Runder Tisch Sexueller Kindesmissbrauch in Abhängigkeits- und Machtverhältnissen und privaten und öffentlichen Einrichtungen und im familiären Bereich. Verfügbar unter www.bmfsfj.de/blob/ 93204/2a2c26eb1dd477abc63a6025bb1b24b9/abschlussbe richt-runder-tisch-sexueller-kindesmissbrauch-data.pdf

Enders, U. \& Schlingmann, T. (2018). Nachhaltige Aufarbeitung aktueller Fälle sexuellen Missbrauchs. In J. Fegert, M. Kölch, E. König, D. Harsch, S. Witte \& U. Hoffmann (Hrsg.), Schutz vor sexueller Gewalt und Übergriffen in Institutionen - Für die Lei- 
tungspraxis im Gesundheitswesen, Jugendhilfe und Schule (S. 299 -311). Berlin: Springer.

Ethik-Kommission der 3 Fachgesellschaften für Kinder- und Jugendpsychiatrie und Psychotherapie (2004). Ärztlichethische Leitlinie zur körperlichen und neurologischen Untersuchung in der Kinder- und Jugendpsychiatrie und -psychotherapie. Verfügbar unter https://www.kinderpsychiater. org/fileadmin/downloads/ll-ku.pdf

Fegert, J. M., Allroggen, M. \& Schloz, C. (2017). Besonderheiten bei der Umsetzung von Schutzkonzepten in Kliniken. In M. Wolff, W. Schroer \& J. M. Fegert (Hrsg.), Schutzkonzepte in Theorie und Praxis. Ein beteiligungsorientiertes Werkbuch (S. 228-232). Weinheim: BeltzJuventa.

Fegert, J. M., Hoffmann, U. \& König, E. (2020). Institutionelle Schutzkonzepte zur Prävention sexuellen Kindesmissbrauchs. In M. Kölch, M. Rassenhofer \& J. M. Fegert (2020). Klinikmanual Kinder- und Jugendpsychiatrie und psychotherapie (S. 669682). Berlin: Springer.

Fegert, J. M., Rassenhofer, M., Schneider, T., Spröber N. \& Seitz, A. (Hrsg.). (2013). Sexueller Kindesmissbrauch - Zeugnisse, Botschaften, Konsequenzen - Ergebnisse der Begleitforschung für die Anlaufstelle der Unabhängigen Beauftragten der Bundesregierung zur Aufarbeitung des sexuellen Kindesmissbrauchs, Frau Dr. Christine Bergmann. Weinheim und Basel: Beltz Juventa.

Fegert, J., Hoffmann, U. \& Kölch, M. (2018). Schutz von vulnerablen Personen im Abhängigkeitsverhältnis. In J. Fegert, M. Kölch, E. König, D. Harsch, S. Witte \& U. Hoffmann (Hrsg.), Schutz vor sexueller Gewalt und Übergriffen in Institutionen Für die Leitungspraxis im Gesundheitswesen, Jugendhilfe und Schule (S. 349-360). Berlin: Springer.

Fegert, J., Kölch, M. \& Kliemann, A. (2018). Kinderschutz in Institutionen - eine Einführung. In J. Fegert, M. Kölch, E. König, D. Harsch, S. Witte \& U. Hoffmann (2018), Schutz vor sexueller Gewalt und Übergriffen in Institutionen - Für die Leitungspraxis im Gesundheitswesen, Jugendhilfe und Schule (S. 3-10). Berlin: Springer.

Fegert, J., Kölch, M., König, E., Harsch, D., Witte, S. \& Hoffmann, U. (2018). Schutz vor sexueller Gewalt und Übergriffen in Institutionen - Für die Leitungspraxis im Gesundheitswesen, Jugendhilfe und Schule. Berlin: Springer.

Fegert, J., Schröer, W. \& Wolff, M. (2017): Persönliche Rechte von Kindern und Jugendlichen. Schutzkonzepte als organisationale Herausforderungen. In M. Wolff, W. Schröer \& J. Fegert (Hrsg.), Schutzkonzepte in Theorie und Praxis. Ein beteiligungsorientiertes Werkbuch (S. 14-24). Weinheim \& Basel: BeltzJuventa.

Fegert, J.M. \& Wolff, M. (Hrsg.). (2002). Sexueller Missbrauch durch Professionelle in Institutionen. Prävention und Intervention. Ein Werkbuch. Münster: Votum.

Fegert, J.M. \& Wolff, M. (Hrsg.). (2006). Sexueller Missbrauch durch Professionelle in Institutionen. Prävention und Intervention. Ein Werkbuch (2. Aufl.). Münster: Votum.

G-BA (2020). Beschluss des Gemeinsamen Bundesausschusses über eine Änderung der Qualitätsmanagement-Richtlinie (QM-RL): Vorgaben für die regelmäßige Erhebung und Darlegung des aktuellen Stands der Umsetzung und Weiterentwicklung von einrichtungsinternem Qualitätsmanagement sowie weitere Änderung in \$ 4 der Richtlinie. https://www.g-ba.de/downloads/39-261-4379/202007-16_QM-RL_Vorgaben-aktueller-Stand.pdf
Hoffmann, U. \& Fegert, J. (2018). Schutz von Kindern und Jugendlichen vor Gewalt in der Pflege. In J. Fegert, M. Kölch, E. König, D. Harsch, S. Witte \& U. Hoffmann (Hrsg.), Schutz vor sexueller Gewalt und Übergriffen in Institutionen - Für die Leitungspraxis im Gesundheitswesen, Jugendhilfe und Schule (S. 383 - 392). Berlin: Springer.

Horvay, R. \& Naumann, A. (2018). Schutz vor grenzverletzendem Verhalten und Übergriffen in einer Klinik für Kinder- und Jugendpsychiatrie. Konzept zur Prävention, Intervention und Aufarbeitung. Norderstedt: BoD, Books on Demand.

Kappler, S., Hornfeck, F., Pooch, M. T., Kindler, H. \& Tremel, I. (2019). Kinder und Jugendliche besser schützen - der Anfang ist gemacht. Schutzkonzepte gegen sexuelle Gewalt in den Bereichen: Bildung und Erziehung, Gesundheit, Freizeit. https://www. dji.de/fileadmin/user_upload/bibs2019/28116_UBSKM_D Jl_Abschlussbericht.pdf

Kölch, M. \& Fegert J. (2018a). Herausforderungen für Leitungskräfte beim Kinderschutz in Institutionen. In J. Fegert, M. Kölch, E. König, D. Harsch, S. Witte \& U. Hoffmann (Hrsg.), Schutz vor sexueller Gewalt und Übergriffen in Institutionen - Für die Leitungspraxis im Gesundheitswesen, Jugendhilfe und Schule (S. $11-18)$. Berlin: Springer.

Kölch, M. \& Fegert J. (2018b). Umgang mit Fehlverhalten und Verdachtsfällen. In J. Fegert, M. Kölch, E. König, D. Harsch, S. Witte \& U. Hoffmann (Hrsg.), Schutz vor sexueller Gewalt und Übergriffen in Institutionen - Für die Leitungspraxis im Gesundheitswesen, Jugendhilfe und Schule (S. 231-239). Berlin: Springer.

König, E., Hoffmann, U., Witte, S., Harsch, D., Kölch, M. \& Fegert J. (2018). Anwendungsbereich für den Transfer in die Praxis. In J. Fegert, M. Kölch, E. König, D. Harsch, S. Witte \& U. Hoffmann (Hrsg.), Schutz vor sexueller Gewalt und Übergriffen in Institutionen - Für die Leitungspraxis im Gesundheitswesen, Jugendhilfe und Schule (S. 443-541). Berlin: Springer.

Rau, T. \& Liebhardt, H. (2018). Partizipationsmöglichkeiten und Beschwerdemanagement. In J. Fegert, M. Kölch, E. König, D. Harsch, S. Witte \& U. Hoffmann (Hrsg.), Schutz vor sexueller Gewalt und Übergriffen in Institutionen - Für die Leitungspraxis im Gesundheitswesen, Jugendhilfe und Schule (S. 217-227). Berlin: Springer.

UBSKM (2021). Schutzkonzepte. https://beauftragter-miss brauch.de/praevention/schutzkonzepte/

Universitätsklinikums des Saarlandes (2020). Schutzkonzept Gewaltprävention am UKS. https://www.uniklinikum-saarland.de/ wir_ueber_uns/schutzkonzept_gewaltpraevention_am_uks/

Winter, V. \& Wolff, M. (2018). Intervention. In J. Fegert, M. Kölch, E. König, D. Harsch, S. Witte \& U. Hoffmann (Hrsg.), Schutz vor sexueller Gewalt und Übergriffen in Institutionen - Für die Leitungspraxis im Gesundheitswesen, Jugendhilfe und Schule (S. $241-250)$. Berlin: Springer.

\section{Dr. Ulrike Hoffmann}

Klinik für Kinder- und Jugendpsychiatrie/Psychotherapie Universitätsklinikum Ulm

Steinhövelstraße 5

$89073 \mathrm{Ulm}$

ulrike.hoffmann@uniklinik-ulm.de 